Теорія Ймовір. та Матем. Статист. Вип. 82, 2010
Theor. Probability and Math. Statist.

No. 82, 2011, Pages 115-127

S 0094-9000(2011)00831-9

Article electronically published on August 4, 2011

\title{
APPROXIMATION OF MULTIFRACTIONAL BROWNIAN MOTION BY ABSOLUTELY CONTINUOUS PROCESSES
}

UDC 519.21

\author{
K. V. RAL'CHENKO
}

\begin{abstract}
We consider absolutely continuous stochastic processes that converge to multifractional Brownian motion in Besov-type spaces. We prove that solutions of stochastic differential equations with these processes converge to the solution of the equation with multifractional Brownian motion.
\end{abstract}

\section{INTRODUCTION}

Fractional Brownian motion is a popular model of randomness in studies of various phenomena in nature, computer nets, financial markets, etc. Fractional Brownian motion is defined as a Gaussian process $B_{t}^{H}$ with zero mean, $\mathrm{E} B_{t}^{H}=0$, and covariance function

$$
\mathrm{E}\left(B_{t}^{H} B_{s}^{H}\right)=\frac{1}{2}\left(t^{2 H}+s^{2 H}-|t-s|^{2 H}\right),
$$

where $H \in(0,1)$ is the so-called Hurst parameter. It is known that if $H \in\left(\frac{1}{2}, 1\right)$, then fractional Brownian motion possesses the so-called long memory property. Fractional Brownian motion $B_{t}^{H}$ is used in such a case to model many processes with long range dependence.

Since increments of fractional Brownian motion are homogeneous, the area of its possible applications is rather restricted. In particular, stochastic processes whose path regularity and "memory depth" evolve in time do not fit the model of fractional Brownian motion. Thus several authors recently provided different generalizations of fractional Brownian motion by assuming that the Hurst parameter $H$ depends on the time $t$ (see 2 , [3, 4, 8, 9]).

We consider the problem of approximation of multifractional Brownian motion by absolutely continuous stochastic processes. We construct approximations and prove that they converge to fractional Brownian motion. We also prove the convergence of solutions of the corresponding stochastic differential equations.

The paper is organized as follows. We recall necessary definitions and results in Section 2. We also discuss several generalizations of multifractional Brownian motion. In Section 3, we introduce certain approximations of multifractional Brownian motion by absolutely continuous processes and prove that they converge. In Section 4 , we consider stochastic differential equations with multifractional Brownian motion. For such equations, we prove the existence, uniqueness, and integrability of a solution. We also

2010 Mathematics Subject Classification. Primary 60G15; Secondary 60H10, 65C30.

Key words and phrases. Gaussian process, fractional Brownian motion, multifractional Brownian motion, stochastic differential equation, Young integral.

The first author is indebted to the European Commission for support in the framework of the "Marie Curie Actions" program, grant PIRSES-GA-2008-230804. 
construct approximations for the solution based on the approximations considered in Section 3. We also prove the convergence of these approximations.

\section{Definitions}

Let $W_{0}^{\beta}=W_{0}^{\beta}[0, T], \beta \in(0,1)$, be the space of measurable functions $f:[0, T] \rightarrow \mathbb{R}$ such that

$$
\|f\|_{0, \beta}:=\sup _{t \in[0, T]}\left(|f(t)|+\int_{0}^{t} \frac{|f(t)-f(s)|}{(t-s)^{1+\beta}} d s\right)<\infty .
$$

Also let $W_{1}^{\beta}=W_{1}^{\beta}[0, T], \beta \in(0,1)$, be the space of measurable functions $f:[0, T] \rightarrow \mathbb{R}$ such that

$$
\|f\|_{1, \beta}:=\sup _{0 \leq s<t \leq T}\left(\frac{|f(t)-f(s)|}{(t-s)^{\beta}}+\int_{s}^{t} \frac{|f(u)-f(s)|}{(u-s)^{1+\beta}} d u\right)<\infty .
$$

We also consider the norm

$$
\|f\|_{0, \beta, \lambda}:=\sup _{t \in[0, T]}\left(e^{-\lambda t}\left[|f(t)|+\int_{0}^{t} \frac{|f(t)-f(s)|}{(t-s)^{1+\beta}} d s\right]\right)
$$

in the space $W_{0}^{\beta}[0, T]$. Note that this norm is equivalent to $\|\cdot\|_{0, \beta}$.

Let $f \in L^{1}(a, b)$ and $\alpha>0$. The left and right sided fractional Riemann-Liouville integrals of order $\alpha$ are defined for a function $f$ almost surely and for almost all $x \in(a, b)$ by the following equalities:

$$
I_{a+}^{\alpha} f(x):=\frac{1}{\Gamma(\alpha)} \int_{a}^{x}(x-y)^{\alpha-1} f(y) d y
$$

and

$$
I_{b-}^{\alpha} f(x):=\frac{(-1)^{-\alpha}}{\Gamma(\alpha)} \int_{x}^{b}(y-x)^{\alpha-1} f(y) d y
$$

respectively (see Definition 2.1 in [10]), where $(-1)^{-\alpha}=e^{-i \pi \alpha}$ and where

$$
\Gamma(\alpha)=\int_{0}^{\infty} r^{\alpha-1} e^{-r} d r
$$

is the Euler Gamma function.

The images of the space $L^{p}(a, b)$ under the actions of the operators $I_{a+}^{\alpha}$ and $I_{b-}^{\alpha}$ are denoted by $I_{a+}^{\alpha}\left(L^{p}\right)$ and $I_{b-}^{\alpha}\left(L^{p}\right)$, respectively. If $0<\alpha<1$ and if $f \in I_{a+}^{\alpha}\left(L^{p}\right)$ or if $f \in I_{b-}^{\alpha}\left(L^{p}\right)$, then the Weil fractional derivatives

$$
D_{a+}^{\alpha} f(x)=\frac{1}{\Gamma(1-\alpha)}\left(\frac{f(x)}{(x-a)^{\alpha}}+\alpha \int_{a}^{x} \frac{f(x)-f(y)}{(x-y)^{\alpha+1}} d y\right) \mathbb{1}_{(a, b)}(x)
$$

or, respectively,

$$
D_{b-}^{\alpha} f(x)=\frac{(-1)^{\alpha}}{\Gamma(1-\alpha)}\left(\frac{f(x)}{(b-x)^{\alpha}}+\alpha \int_{x}^{b} \frac{f(x)-f(y)}{(y-x)^{\alpha+1}} d y\right) \mathbb{1}_{(a, b)}(x)
$$

are defined for almost all $x \in(a, b)$.

If the limits $f(a+)$ and $g(b-)$ exist and are finite, then we put

$$
\begin{aligned}
f_{a+}(x) & =(f(x)-f(a+)) \mathbb{1}_{(a, b)}(x), \\
g_{b-}(x) & =(g(x)-g(b-)) \mathbb{1}_{(a, b)}(x) .
\end{aligned}
$$

Let $(\Omega, \mathcal{F}, \mathrm{P})$ be a complete probability space. 
Definition 2.1. Let $H \in(0,1)$. A centered Gaussian process $B^{H}=\left\{B_{t}^{H}, t \geq 0\right\}$ with stationary increments and covariance function

$$
\mathrm{E}\left(B_{t}^{H} B_{s}^{H}\right)=\frac{1}{2}\left(t^{2 H}+s^{2 H}-|t-s|^{2 H}\right)
$$

is called a fractional Brownian motion with the Hurst parameter $H$.

Assume that the function $H:[0,+\infty) \rightarrow\left(\frac{1}{2}, 1\right)$ satisfies the Hölder condition with index $\gamma>\frac{1}{2}$, namely,

$$
\left|H_{t_{1}}-H_{t_{2}}\right| \leq C_{1}\left|t_{1}-t_{2}\right|^{\gamma}
$$

for all $t_{1}, t_{2} \in[0,+\infty)$ and some $C_{1}>0$.

There are several generalizations of fractional Brownian motion to the case where the Hurst index $H$ is varying with time.

Example 1. Multifractional Brownian motion is introduced in $[8$. The definition in $[8]$ is based on the Mandelbrot-Van Ness representation for multifractional Brownian motion (see, for example, [6, Chapter 1.3]). According to [8], fractional Brownian motion is defined by $Y_{t}=B_{t}^{H_{t}}, t \geq 0$, where $H \in\left(\frac{1}{2}, 1\right)$,

$$
B_{t}^{H}=\frac{1}{\Gamma\left(H+\frac{1}{2}\right)}\left\{\int_{-\infty}^{0}\left[(t-s)^{H-1 / 2}-(-s)^{H-1 / 2}\right] d W_{s}+\int_{0}^{t}(t-s)^{H-1 / 2} d W_{s}\right\},
$$

and where $W_{s}$ is a Wiener process.

Example 2. In the papers [3, 9], the authors define the so-called multifractional Volterratype Brownian motion based on the representation of the fractional Brownian motion in terms of the Molchan martingale (see, for example, [6, Section 1.8]). The multifractional Volterra-type Brownian motion in the process $Y_{t}=B_{t}^{H_{t}}$, where

$$
B_{t}^{H}=\int_{0}^{t} K_{H}(t, s) d W_{s}, \quad t \geq 0,
$$

$W_{s}$ is a Wiener process, and where

$$
\begin{gathered}
K_{H}(t, s)=C_{H} s^{1 / 2-H} \int_{s}^{t}(v-s)^{H-3 / 2} v^{H-1 / 2} d v, \\
C_{H}=\left(\frac{H(2 H-1)}{\mathrm{B}\left(2-2 H, H-\frac{1}{2}\right)}\right)^{1 / 2} .
\end{gathered}
$$

Example 3. Consider another generalization, called the harmonizable multifractional Brownian motion (see, for example, 2, 4]). Let $W(\cdot)$ be a complex random measure on $\mathbb{R}$ such that

1) for all $A, B \in \mathcal{B}(\mathbb{R})$,

$$
\mathrm{E} W(A) \overline{W(B)}=\lambda(A \cap B),
$$

where $\lambda$ is the Lebesgue measure;

2) for an arbitrary sequence $\left\{A_{1}, A_{2}, \ldots\right\} \subset \mathcal{B}(\mathbb{R})$ such that $A_{i} \cap A_{j}=\varnothing$ for all $i \neq j$, we have

$$
W\left(\bigcup_{i \geq 1} A_{i}\right)=\sum_{i \geq 1} W\left(A_{i}\right)
$$

(here $\left\{W\left(A_{i}\right), i \geq 1\right\}$ are centered normal random variables);

3) for all $A \in \mathcal{B}(\mathbb{R})$,

$$
W(A)=\overline{W(-A)}
$$


4) for all $\theta \in \mathbb{R}$,

$$
\left\{e^{i \theta} W(A), A \in \mathcal{B}(\mathbb{R})\right\} \stackrel{d}{=}\{W(A), A \in \mathcal{B}(\mathbb{R})\} .
$$

We define the multifractional Brownian motion in this case by $Y_{t}=B_{t}^{H_{t}}$, where

$$
B_{t}^{H}=\int_{\mathbb{R}} \frac{e^{i t x}-1}{|x|^{1 / 2+H}} W(d x) .
$$

In what follows we consider one more generalization of the fractional Brownian motion defined by $Y_{t}=B_{t}^{H_{t}}$, where $\left\{B_{t}^{H}, t \in[0, T], H \in\left(\frac{1}{2}, 1\right)\right\}$ is a family of random variables such that

(i) for a fixed $H \in\left(\frac{1}{2}, 1\right)$, the process $\left\{B_{t}^{H}, t \in[0, T]\right\}$ is a fractional Brownian motion with the Hurst parameter $H$;

(ii) for all $t \in[0, T]$,

$$
\mathrm{E}\left(B_{t}^{H_{1}}-B_{t}^{H_{2}}\right)^{2} \leq C_{2}(\delta)\left(H_{1}-H_{2}\right)^{2},
$$

where $H_{1}, H_{2} \in\left[\frac{1}{2}+\delta, 1-\delta\right]$ for some $\delta \in\left(0, \frac{1}{4}\right)$ and where $C_{2}(\delta)$ is a constant.

All the above conditions are satisfied, for instance, by every one of the generalizations described in Examples 133, since conditions (ii) and (iii) hold for representations (2.2)(2.4) (see [4, 8, 9]).

In what follows $\delta$ is fixed and equal to

$$
\max \left\{\min _{t \in[0, T]} H_{t}-\frac{1}{2}, 1-\max _{t \in[0, T]} H_{t}\right\} .
$$

We denote $C_{2}=C_{2}(\delta)$ and $H_{\min }:=\min \left\{\gamma, \min _{t \in[0, T]} H_{t}\right\}$.

Remark. Conditions (ii) and (iii) imply that the trajectories of the process $Y_{t}=B_{t}^{H_{t}}$ are continuous almost surely. Indeed, the process $B_{t}^{H_{t}}$ with $H_{t}=$ const is a fractional Brownian motion and thus

$$
\mathrm{E}\left(B_{t}^{H_{t}}-B_{s}^{H_{t}}\right)^{2}=|t-s|^{2 H_{t}}
$$

for all $s \in[0, T]$ and $t \in[0, T]$. Now we use inequalities (2.1) and (2.5) and obtain the following bound for the second moment

$$
\begin{aligned}
\mathrm{E}\left(Y_{t}-Y_{s}\right)^{2} & \leq 2\left(\mathrm{E}\left(B_{t}^{H_{t}}-B_{s}^{H_{t}}\right)^{2}+\mathrm{E}\left(B_{s}^{H_{t}}-B_{s}^{H_{s}}\right)^{2}\right) \\
& \leq 2\left(|t-s|^{2 H_{t}}+C_{1}^{2} C_{2}|t-s|^{2 \gamma}\right) .
\end{aligned}
$$

Note that it is sufficient to show that the process $Y_{t}$ is continuous on every interval $[a, b] \subset[0, T]$ such that $b-a<1$. Hence we can assume $|t-s|<1$ without loss of generality. Then

$$
\mathrm{E}\left(Y_{t}-Y_{s}\right)^{2} \leq 2\left(1+C_{1}^{2} C_{2}\right)|t-s|^{2 H_{\min }} .
$$

Since $Y_{t}$ is a Gaussian process, the latter bound implies that, given an arbitrary $r>0$, there exists $C>0$ such that

$$
\mathrm{E}\left(\left|Y_{t}-Y_{s}\right|^{r}\right) \leq C|t-s|^{r H_{\min }}
$$

for all $t, s \in[0, T]$. By the Kolmogorov criterion, this means that the process $Y_{t}$ is continuous almost surely. 


\section{An APproximation of MULTIFRACTIONAL BROWNiAN MOTION}

Consider the following approximation:

$$
B_{t}^{H_{t}, \varepsilon}:=\frac{1}{\phi_{t}(\varepsilon)} \int_{t}^{t+\phi_{t}(\varepsilon)} B_{s}^{H_{s}} d s=\frac{1}{\phi_{t}(\varepsilon)} \int_{0}^{\phi_{t}(\varepsilon)} B_{u+t}^{H_{u+t}} d u
$$

for the fractional Brownian motion, where $\phi_{t}(\varepsilon)=\phi(t, \varepsilon):[0, T] \times \mathbb{R}_{+} \rightarrow \mathbb{R}_{+}$is a family of measurable functions such that

1) $f_{\varepsilon}:=\sup _{t \in[0, T]} \phi_{t}(\varepsilon) \rightarrow 0$ as $\varepsilon \rightarrow 0+$;

2) for all $t, s \in[0, T]$ and for all $\varepsilon>0$,

$$
\left|\frac{\phi_{s}(\varepsilon)-\phi_{t}(\varepsilon)}{\phi_{s}(\varepsilon)}\right| \leq C_{3}|t-s|^{H_{\min }}
$$

Here $C_{3}$ is a constant that does not depend on $\varepsilon$.

One can take, for example, $\phi_{t}(\varepsilon)=\psi_{t} \cdot \varepsilon$, where $\psi_{t}$ is bounded away from zero and satisfies the Hölder condition with index $H_{\min }$.

Theorem 3.1. For an arbitrary $\beta \in\left(0, H_{\min }\right)$,

$$
\left\|B^{H, \varepsilon}-B^{H}\right\|_{1, \beta} \stackrel{\mathrm{P}}{\rightarrow} 0, \quad \varepsilon \rightarrow 0+.
$$

Proof. To make expressions simpler we put $Y_{t}=B_{t}^{H_{t}}$ and $Y_{t}^{\varepsilon}=B_{t}^{H_{t}, \varepsilon}$.

It is easy to see that

$$
\Delta Y_{t}^{\varepsilon}:=Y_{t}^{\varepsilon}-Y_{t}=\frac{1}{\phi_{t}(\varepsilon)} \int_{0}^{\phi_{t}(\varepsilon)}\left(Y_{u+t}-Y_{t}\right) d u
$$

Now we show that

$$
\mathrm{E}\left(\left(\Delta Y_{t}^{\varepsilon}-\Delta Y_{s}^{\varepsilon}\right)^{2}\right) \leq|t-s|^{H_{\min }+\beta} g_{\varepsilon}
$$

where the function $g_{\varepsilon}$ is such that $g_{\varepsilon} \rightarrow 0$ as $\varepsilon \rightarrow 0+$. Further, we rewrite the difference $\left(\Delta Y_{t}^{\varepsilon}-\Delta Y_{s}^{\varepsilon}\right)$ in the following form:

$$
\begin{aligned}
\Delta Y_{t}^{\varepsilon}-\Delta Y_{s}^{\varepsilon}= & \left(\frac{1}{\phi_{t}(\varepsilon)}-\frac{1}{\phi_{s}(\varepsilon)}\right) \int_{0}^{\phi_{t}(\varepsilon)}\left(Y_{u+t}-Y_{t}\right) d u \\
& +\frac{1}{\phi_{s}(\varepsilon)} \int_{\phi_{s}(\varepsilon)}^{\phi_{t}(\varepsilon)}\left(Y_{u+t}-Y_{t}\right) d u+\frac{1}{\phi_{s}(\varepsilon)} \int_{0}^{\phi_{s}(\varepsilon)}\left(Y_{u+t}-Y_{t}-Y_{u+s}+Y_{s}\right) d u .
\end{aligned}
$$

Then

$$
\begin{aligned}
\mathrm{E}\left(\left(\Delta Y_{t}^{\varepsilon}-\Delta Y_{s}^{\varepsilon}\right)^{2}\right) \leq & 3\left(\left(\frac{\phi_{s}(\varepsilon)-\phi_{t}(\varepsilon)}{\phi_{t}(\varepsilon) \phi_{s}(\varepsilon)}\right)^{2} \mathrm{E}\left(\int_{0}^{\phi_{t}(\varepsilon)}\left(Y_{u+t}-Y_{t}\right) d u\right)^{2}\right. \\
& +\frac{1}{\left(\phi_{s}(\varepsilon)\right)^{2}} \mathrm{E}\left(\int_{\phi_{s}(\varepsilon)}^{\phi_{t}(\varepsilon)}\left(Y_{u+t}-Y_{t}\right) d u\right)^{2} \\
& \left.+\frac{1}{\left(\phi_{s}(\varepsilon)\right)^{2}} \mathrm{E}\left(\int_{0}^{\phi_{s}(\varepsilon)}\left(Y_{u+t}-Y_{t}-Y_{u+s}+Y_{s}\right) d u\right)^{2}\right) \\
= & 3\left(I_{1}+I_{2}+I_{3}\right) .
\end{aligned}
$$

Consider the term $I_{1}$. By the Cauchy-Bunyakovskiǔ inequality,

$$
I_{1} \leq\left(\frac{\phi_{s}(\varepsilon)-\phi_{t}(\varepsilon)}{\phi_{t}(\varepsilon) \phi_{s}(\varepsilon)}\right)^{2} \phi_{t}(\varepsilon) \int_{0}^{\phi_{t}(\varepsilon)} \mathrm{E}\left(Y_{u+t}-Y_{t}\right)^{2} d u \text {. }
$$


Considering relations (2.6) and (3.2) we get

$$
I_{1} \leq \frac{2 C_{3}^{2}|t-s|^{2 H_{\min }}}{\phi_{t}(\varepsilon)} \int_{0}^{\phi_{t}(\varepsilon)}\left(u^{2 H_{u+t}}+C_{1}^{2} C_{2} u^{2 \gamma}\right) d u .
$$

Since $\sup _{t \in[0, T]} \phi_{t}(\varepsilon) \rightarrow 0$, there exists $\varepsilon_{0}>0$ such that

$$
\sup _{t \in[0, T]} \phi_{t}(\varepsilon) \leq 1
$$

for all $\varepsilon \in\left(0, \varepsilon_{0}\right)$. This allows us to consider only the case of $\phi_{t}(\varepsilon) \leq 1$. Then the integrand in (3.4) is estimated as follows:

$$
u^{2 H_{u+t}}+C_{1}^{2} C_{2} u^{2 \gamma} \leq\left(1+C_{1}^{2} C_{2}\right) u^{2 H_{\min }} .
$$

Finally we get

$$
I_{1} \leq \frac{2 C_{3}^{2}\left(1+C_{1}^{2} C_{2}\right)}{2 H_{\min }+1}|t-s|^{2 H_{\min }}\left(\phi_{t}(\varepsilon)\right)^{2 H_{\min }} \leq g_{\varepsilon}^{(1)}|t-s|^{H_{\min }+\beta},
$$

where

$$
g_{\varepsilon}^{(1)}=\frac{2 C_{3}^{2}\left(1+C_{1}^{2} C_{2}\right) T^{H_{\min }-\beta}}{2 H_{\min }+1} f_{\varepsilon}^{2 H_{\min }} \rightarrow 0, \quad \varepsilon \rightarrow 0+.
$$

The term $I_{2}$ is estimated similarly:

$$
\begin{aligned}
I_{2} & \leq \frac{\phi_{t}(\varepsilon)-\phi_{s}(\varepsilon)}{\left(\phi_{s}(\varepsilon)\right)^{2}} \int_{\phi_{s}(\varepsilon)}^{\phi_{t}(\varepsilon)} \mathrm{E}\left(Y_{u+t}-Y_{t}\right)^{2} d u \\
& \leq \frac{2\left(\phi_{t}(\varepsilon)-\phi_{s}(\varepsilon)\right)}{\left(\phi_{s}(\varepsilon)\right)^{2}} \int_{\phi_{s}(\varepsilon)}^{\phi_{t}(\varepsilon)}\left(u^{2 H_{u+t}}+C_{1}^{2} C_{2} u^{2 \gamma}\right)^{2} d u .
\end{aligned}
$$

The expression under the integral sign is estimated by

$$
u^{2 H_{u+t}}+C_{1}^{2} C_{2} u^{2 \gamma} \leq\left(1+C_{1}^{2} C_{2}\right) u^{2 H_{\min }} \leq\left(1+C_{1}^{2} C_{2}\right) f_{\varepsilon}^{2 H_{\min }} .
$$

Taking into account bound (3.2) we prove that

$$
I_{2} \leq 2 C_{3}^{2}\left(1+C_{1}^{2} C_{2}\right)|t-s|^{2 H_{\min }} f_{\varepsilon}^{2 H_{\min }} \leq g_{\varepsilon}^{(2)}|t-s|^{H_{\min }+\beta},
$$

where

$$
g_{\varepsilon}^{(2)}=2 C_{3}^{2}\left(1+C_{1}^{2} C_{2}\right) T^{H_{\min }-\beta} f_{\varepsilon}^{2 H_{\min }} \rightarrow 0, \quad \varepsilon \rightarrow 0+.
$$

Now we estimate the term $I_{3}$ :

$$
I_{3} \leq \frac{1}{\phi_{s}(\varepsilon)} \int_{0}^{\phi_{s}(\varepsilon)} \mathrm{E}\left(Y_{u+t}-Y_{t}-Y_{u+s}+Y_{s}\right)^{2} d u
$$

by the Cauchy-Bunyakovskil inequality.

Further we consider two possible cases.

Case 1. $\phi_{s}(\varepsilon) \leq|t-s|$. Then

$$
\begin{aligned}
I_{3} & \leq \frac{2}{\phi_{s}(\varepsilon)} \int_{0}^{\phi_{s}(\varepsilon)}\left(\mathrm{E}\left(Y_{u+t}-Y_{t}\right)^{2}+\mathrm{E}\left(Y_{u+s}-Y_{s}\right)^{2}\right) d u \\
& \leq \frac{4}{\phi_{s}(\varepsilon)} \int_{0}^{\phi_{s}(\varepsilon)}\left(u^{2 H_{u+t}}+u^{2 H_{u+s}}+2 C_{1}^{2} C_{2} u^{2 \gamma}\right) d u \\
& \leq \frac{8\left(1+C_{1}^{2} C_{2}\right)}{\phi_{s}(\varepsilon)} \int_{0}^{\phi_{s}(\varepsilon)} u^{2 H_{\min }} d u=\frac{8\left(1+C_{1}^{2} C_{2}\right)}{2 H_{\min }+1}\left(\phi_{s}(\varepsilon)\right)^{2 H_{\min }} \\
& \leq \frac{8\left(1+C_{1}^{2} C_{2}\right)}{2 H_{\min }+1}|t-s|^{H_{\min }+\beta}\left(\phi_{s}(\varepsilon)\right)^{H_{\min }-\beta} \leq g_{\varepsilon}^{(3)}|t-s|^{H_{\min }+\beta},
\end{aligned}
$$


where

$$
g_{\varepsilon}^{(3)}=\frac{8\left(1+C_{1}^{2} C_{2}\right)}{2 H_{\min }+1} f_{\varepsilon}^{H_{\min }-\beta} \rightarrow 0, \quad \varepsilon \rightarrow 0+.
$$

Case 2. $\phi_{s}(\varepsilon)>|t-s|$. Then

$$
\begin{aligned}
I_{3} & \leq \frac{2}{\phi_{s}(\varepsilon)} \int_{0}^{\phi_{s}(\varepsilon)}\left(\mathrm{E}\left(Y_{u+t}-Y_{u+s}\right)^{2}+\mathrm{E}\left(Y_{t}-Y_{s}\right)^{2}\right) d u \\
& \leq \frac{4}{\phi_{s}(\varepsilon)} \int_{0}^{\phi_{s}(\varepsilon)}\left(|t-s|^{2 H_{u+t}}+|t-s|^{2 H_{t}}+2 C_{1}^{2} C_{2}|t-s|^{2 \gamma}\right) d u .
\end{aligned}
$$

Using the estimate

$$
|t-s|^{2 H_{u+t}} \leq T^{2 H_{u+t}}\left|\frac{t-s}{T}\right|^{2 H_{\min }} \leq \max \{T, 1\}|t-s|^{2 H_{\min }}
$$

and similar bounds for $|t-s|^{2 H_{t}}$ and $|t-s|^{2 \gamma}$, we get

$$
I_{3} \leq 8\left(1+C_{1}^{2} C_{2}\right) \max \{T, 1\}|t-s|^{2 H_{\min }} \leq g_{\varepsilon}^{(4)}|t-s|^{H_{\min }+\beta},
$$

where

$$
g_{\varepsilon}^{(4)}=8\left(1+C_{1}^{2} C_{2}\right) \max \{T, 1\} f_{\varepsilon}^{H_{\min }-\beta} \rightarrow 0, \quad \varepsilon \rightarrow 0+.
$$

Therefore inequality (3.3) holds for

$$
g_{\varepsilon}=3\left(g_{\varepsilon}^{(1)}+g_{\varepsilon}^{(2)}+g_{\varepsilon}^{(3)}+g_{\varepsilon}^{(4)}\right) \rightarrow 0, \quad \varepsilon \rightarrow 0+.
$$

Since the random variable $\left(\Delta Y_{t}^{\varepsilon}-\Delta Y_{s}^{\varepsilon}\right)$ has a normal distribution, inequality (3.3) implies that

$$
\mathrm{E}\left(\left|\Delta Y_{t}^{\varepsilon}-\Delta Y_{s}^{\varepsilon}\right|^{p}\right) \leq C_{p}^{\prime}|t-s|^{p\left(H_{\min }+\beta\right) / 2} g_{\varepsilon}^{p / 2}
$$

for all $p>0$.

By Lemma 5.1 ,

$$
\left|\Delta Y_{t}^{\varepsilon}-\Delta Y_{s}^{\varepsilon}\right|^{p} \leq C_{\alpha, p}|t-s|^{\alpha p-1} \xi
$$

for all $p>0, \alpha>\frac{1}{p}$, and $s, t \in[0, T]$, where $C_{\alpha, p}>0$ is a nonrandom constant and where

$$
\xi=\int_{0}^{T} \int_{0}^{T} \frac{\left|\Delta Y_{x}^{\varepsilon}-\Delta Y_{y}^{\varepsilon}\right|^{p}}{|x-y|^{\alpha p+1}} d x d y .
$$

Fix $\delta \in\left(\beta,\left(H_{\min }+\beta\right) / 2\right)$ and consider $p=4 /\left(H_{\min }+\beta-2 \delta\right)$ and $\alpha=\left(H_{\min }+\beta+2 \delta\right) / 4$. Using estimate (3.5) we obtain

$$
\begin{aligned}
\mathrm{E} \xi & =\int_{0}^{T} \int_{0}^{T} \frac{\mathrm{E}\left(\left|\Delta Y_{x}^{\varepsilon}-\Delta Y_{y}^{\varepsilon}\right|^{p}\right)}{|x-y|^{\alpha p+1}} d x d y \\
& \leq C_{p}^{\prime} g_{\varepsilon}^{p / 2} \int_{0}^{T} \int_{0}^{T}|x-y|^{p\left(H_{\min }+\beta\right) / 2-\alpha p-1} d x d y=C_{p}^{\prime} T^{2} g_{\varepsilon}^{p / 2} .
\end{aligned}
$$

Thus we deduce from (3.6) that

$$
\mathrm{E} \sup _{s, t \in[0, T]} \frac{\left|\Delta Y_{t}^{\varepsilon}-\Delta Y_{s}^{\varepsilon}\right|^{p}}{|t-s|^{\delta p}} \leq C_{\alpha, p} C_{p}^{\prime} T^{2} g_{\varepsilon}^{p / 2} .
$$

The latter bound means that, given an arbitrary $\kappa \in(0,1)$, there exists a constant $C_{\kappa}$ such that the probability of the random event

$$
A_{\varepsilon}:=\left\{\left|\Delta Y_{t}^{\varepsilon}-\Delta Y_{s}^{\varepsilon}\right| \leq C_{\kappa}|t-s|^{\delta} g_{\varepsilon}^{1 / 2} \text { for all } s, t \in[0, T]\right\}
$$

is not less than $1-\kappa$. 
On the random event $A_{\varepsilon}$, we have

$$
\begin{aligned}
\left\|Y^{\varepsilon}-Y\right\|_{1, \beta} & \leq \sup _{0 \leq s<t \leq T}\left(C_{\kappa}|t-s|^{\delta-\beta} g_{\varepsilon}^{1 / 2}+\int_{s}^{t} C_{\kappa}|u-s|^{\delta-1-\beta} g_{\varepsilon}^{1 / 2} d u\right) \\
& =\sup _{0 \leq s<t \leq T}\left(C_{\kappa} g_{\varepsilon}^{1 / 2}|t-s|^{\delta-\beta}\left(1+(\delta-\beta)^{-1}\right)\right) \\
& \leq C_{\kappa} g_{\varepsilon}^{1 / 2} T^{\delta-\beta}\left(1+(\delta-\beta)^{-1}\right) \rightarrow 0, \quad \varepsilon \rightarrow 0+.
\end{aligned}
$$

Then, for an arbitrary $a>0$,

$$
\varlimsup_{\varepsilon \rightarrow 0+} \mathrm{P}\left(\left\|Y^{\varepsilon}-Y\right\|_{1, \beta} \geq a\right) \leq \kappa,
$$

since $C_{\kappa} g_{\varepsilon}^{1 / 2} T^{\delta-\beta}\left(1+(\delta-\beta)^{-1}\right)<a$ for all sufficiently small $\varepsilon$.

Therefore if $\kappa \rightarrow 0+$, then

$$
\lim _{\varepsilon \rightarrow 0+} \mathrm{P}\left(\left\|Y^{\varepsilon}-Y\right\|_{1, \beta} \geq a\right)=0,
$$

and this completes the proof.

Note that the case of $H_{t}=H=$ const corresponds to the usual fractional Brownian motion.

Corollary 3.1. Let $\left\{B_{t}^{H}, t \in[0, T]\right\}$ be a fractional Brownian motion with the Hurst parameter $H \in\left(\frac{1}{2}, 1\right)$ and let the functions $\phi_{t}(\varepsilon)$ satisfy the following conditions:

1) $\sup _{t \in[0, T]} \phi_{t}(\varepsilon) \rightarrow 0$ as $\varepsilon \rightarrow 0+$;

2) for all $t, s \in[0, T]$ and for all $\varepsilon>0$,

$$
\left|\frac{\phi_{s}(\varepsilon)-\phi_{t}(\varepsilon)}{\phi_{s}(\varepsilon)}\right| \leq C_{3}|t-s|^{H}
$$

where $C_{3}$ is a constant that does not depend on $\varepsilon$.

Then the approximations

$$
B_{t}^{H, \varepsilon}=\frac{1}{\phi_{t}(\varepsilon)} \int_{t}^{t+\phi_{t}(\varepsilon)} B_{s}^{H} d s
$$

converge, namely

$$
\left\|B^{H, \varepsilon}-B^{H}\right\|_{1, \beta} \stackrel{\mathrm{P}}{\rightarrow} 0, \quad \varepsilon \rightarrow 0+,
$$

for all $\beta \in(0, H)$.

\section{Approximation of solutions of stochastic differential equations}

Consider the following stochastic differential equation with multifractional Brownian motion:

$$
X_{t}=X_{0}+\int_{0}^{t} b\left(s, X_{s}\right) d s+\int_{0}^{t} \sigma\left(s, X_{s}\right) d B_{s}^{H_{s}}, \quad t \in[0, T] .
$$

It is natural to construct an approximation for a solution of this equation as a solution of the equation

$$
X_{t}^{\varepsilon}=X_{0}+\int_{0}^{t} b\left(s, X_{s}^{\varepsilon}\right) d s+\int_{0}^{t} \sigma\left(s, X_{s}^{\varepsilon}\right) d B_{s}^{H_{s}, \varepsilon}, \quad t \in[0, T],
$$

where the processes $B_{s}^{H_{s}, \varepsilon}, \varepsilon>0$, are defined by equality (3.1). First we find conditions for the existence and uniqueness of a solution of equation (4.1). 
4.1. Existence, uniqueness, and integrability. In general, the existence and uniqueness of a solution follow from the results of [7].

Assume that the coefficients $b(t, x)$ and $\sigma(t, x)$ satisfy almost surely the following conditions (the constants $L_{N}$ and $M_{N}$ that will appear below as well as the function $b_{0}$ may depend on $\omega)$ :

I. The function $\sigma(t, x)$ is differentiable with respect to $x$; there exist constants

$$
1-H_{\min }<\varkappa \leq 1 \text { and } \frac{1}{H_{\min }}-1<\delta \leq 1,
$$

and, for all $N>0$, there is $M_{N}>0$ such that

(i) for all $x \in \mathbb{R}$ and $t \in[0, T]$,

$$
|\sigma(t, x)-\sigma(t, y)| \leq M_{0}|x-y| ;
$$

(ii) for all $|x|,|y| \leq N$ and $t \in[0, T]$,

$$
\left|\frac{\partial}{\partial x} \sigma(t, x)-\frac{\partial}{\partial x} \sigma(t, y)\right| \leq M_{N}|x-y|^{\delta}
$$

(iii) for all $x \in \mathbb{R}$ and $t, s \in[0, T]$,

$$
|\sigma(t, x)-\sigma(s, x)|+\left|\frac{\partial}{\partial x} \sigma(t, x)-\frac{\partial}{\partial x} \sigma(s, x)\right| \leq M_{0}|t-s|^{\varkappa} .
$$

II. There exists $b_{0} \in L^{\rho}(0, T), \rho \geq 2$, and for every $N>0$ there exists $L_{N}>0$ such that

iv) for all $|x|,|y| \leq N$ and $t \in[0, T]$,

$$
|b(t, x)-b(t, y)| \leq L_{N}|x-y|
$$

v) for all $x \in \mathbb{R}$ and $t \in[0, T]$,

$$
|b(t, x)| \leq L_{0}|x|+b_{0}(t) .
$$

Let

$$
\alpha_{0}=\min \left\{\frac{1}{2}, \varkappa, \frac{\delta}{1+\delta}\right\} .
$$

Theorem 4.1. Assume that $\alpha \in\left(1-H_{\min }, \alpha_{0}\right)$. Let $X_{0}$ be a random variable and let the coefficients of equation (4.1) satisfy conditions (i)-(v) with $\rho \geq 1 / \alpha$. Then a solution $\left\{X_{t}, t \in[0, T]\right\}$ of equation (4.1) exists and is unique. Moreover,

$$
X \in L^{0}\left(\Omega, \mathcal{F}, \mathrm{P}, W_{0}^{\alpha}[0, T]\right)
$$

and its trajectories belong to the space $C^{1-\alpha}[0, T]$.

Proof. Theorem 4.1 follows from [7, Theorem 5.1] since the trajectories of the process $B_{t}^{H_{t}}$ belong to $W_{1}^{\beta}[0, T]$ (by Theorem 3.1).

Theorem 4.2. Assume that $X_{0} \in L^{\infty}(\Omega, \mathcal{F}, \mathrm{P}, \mathbb{R})$. Let the coefficients of equation (4.1) satisfy conditions $(\mathrm{i})-(\mathrm{v})$ and additionally the following condition:

vi) there are constants $\mu \in[0,1]$ and $K_{0}>0$ such that

$$
|\sigma(t, x)| \leq K_{0}\left(1+|x|^{\mu}\right)
$$

for all $x \in \mathbb{R}$ and $t \in[0, T]$.

Also let $\alpha \in\left(1-H_{\min }, \min \left\{\alpha_{0}, \frac{2-\mu}{4}\right\}\right)$ and $\rho \geq 1 / \alpha$. Assume that the constants $M_{N}$, $L_{N}$, and $K_{0}$ as well as the function $b_{0}$ do not depend on $\omega$. Then the solution $X$ of equation (4.1) is such that

$$
\mathrm{E}\|X\|_{0, \alpha}^{p}<\infty
$$

for all $p \geq 1$. 
Proof. Denote $\mathbb{T}=\{(s, t): 0 \leq s<t \leq T\}$. Then, for all pairs $(s, t) \in \mathbb{T}$, the random variables

$$
Z_{(s, t)}=\left(D_{t-}^{1-\alpha} Y_{t-}\right)(s)
$$

are jointly Gaussian. By Lemma 5.3 given below,

$$
\mathrm{E} \sup _{0 \leq s<t \leq T}\left|Z_{(s, t)}\right|<+\infty
$$

whence

$$
\mathrm{P}\left(\sup _{0 \leq s<t \leq T} Z_{(s, t)}<+\infty\right)=1 .
$$

According to [1, Theorem 3.2],

$$
\mathrm{E} \exp \left(\varepsilon \sup _{0 \leq s<t \leq T}\left|Z_{(s, t)}\right|^{2}\right)<+\infty
$$

for sufficiently small $\varepsilon$. This implies that

$$
\mathrm{E} e^{c G^{\varkappa}}<+\infty
$$

for arbitrary $C>0$ and $\varkappa<2$, where

$$
G=\frac{1}{\Gamma(1-\alpha)} \sup _{0 \leq s<t \leq T}\left|D_{t-}^{1-\alpha} Y_{t-}(s)\right| .
$$

Using condition (4.3) and [7, Proposition 5.1], we prove that

$$
\|X\|_{0, \alpha} \leq c_{1} e^{c_{2} G^{\varkappa}}
$$

Thus

$$
\mathrm{E}\|X\|_{0, \alpha}^{p} \leq c_{1} \mathrm{E} e^{p c_{2} G^{\varkappa}}<+\infty
$$

for all $p \geq 1$ if $\varkappa<2$.

4.2. Approximations. We turn back to the approximations of a solution of equation (4.1) by solutions of equations (4.2).

Let approximations $B_{s}^{H_{s}, \varepsilon}, \varepsilon>0$, be defined by equality (3.1).

Theorem 4.3. Let all the assumptions of Theorem 4.1 hold. Then

$$
\sup _{t \in[0, T]}\left|X_{t}-X_{t}^{\varepsilon}\right| \stackrel{\mathrm{P}}{\rightarrow} 0, \quad \varepsilon \rightarrow 0+.
$$

Proof. Theorem 4.3 follows from Theorem 5.1 and Theorem 3.1

\section{Appendices}

Lemma 5.1 (The Garsia-Rodemich-Rumsey inequality). Let $p>0, \alpha>\frac{1}{p}$, and let $f \in C([0, T])$. Then there exists a constant $C_{\alpha, p}>0$ such that

$$
|f(t)-f(s)|^{p} \leq C_{\alpha, p}|t-s|^{\alpha p-1} \int_{0}^{T} \int_{0}^{T} \frac{|f(x)-f(y)|^{p}}{|x-y|^{\alpha p+1}} d x d y
$$

for all $s, t \in[0, T]$.

This result follows from Lemma 1.1 of [5].

Lemma 5.2. For every $0<\varepsilon<H_{\min }$, there exists a random variable $\eta_{\varepsilon}>0$ such that $\mathrm{E}\left(\left|\eta_{\varepsilon}\right|^{q}\right)<+\infty$ for all $q>0$ and

$$
\sup _{s, t \in[0, T]} \frac{\left|Y_{s}-Y_{t}\right|}{|s-t|^{H_{\min }-\varepsilon}} \leq \eta_{\varepsilon} .
$$


Proof. Putting $\alpha=H_{\min }-\varepsilon / 2$ and $p=2 / \varepsilon$ in the Garsia-Rodemich-Rumsey inequality (5.1) we show that

$$
\left|Y_{s}-Y_{t}\right| \leq C_{H_{\min }, \varepsilon}|s-t|^{H_{\min }-\varepsilon} \xi
$$

for all $s, t \in[0, T]$, where

$$
\xi=\left(\int_{0}^{T} \int_{0}^{T} \frac{\left|Y_{x}-Y_{y}\right|^{2 / \varepsilon}}{|x-y|^{2 H_{\min } / \varepsilon}} d x d y\right)^{\varepsilon / 2} .
$$

First we assume that $q>2 / \varepsilon$. Then

$$
\mathrm{E}\left(|\xi|^{q}\right) \leq\left(\int_{0}^{T} \int_{0}^{T} \frac{\left(\mathrm{E}\left(\left|Y_{x}-Y_{y}\right|^{q}\right)\right)^{2 /(q \varepsilon)}}{|x-y|^{2 H_{\min } / \varepsilon}} d x d y\right)^{q \varepsilon / 2} .
$$

Considering bounds (2.6) we obtain that

$$
\mathrm{E}\left(\left|Y_{x}-Y_{y}\right|^{2}\right) \leq c_{T}|x-y|^{2 H_{\min }},
$$

whence

$$
\mathrm{E}\left(\left|Y_{x}-Y_{y}\right|^{q}\right) \leq c_{T, q}|x-y|^{q H_{\min }} .
$$

Then inequality (5.3) implies that

$$
\mathrm{E}\left(|\xi|^{q}\right) \leq c_{T, q} T^{q \varepsilon}<+\infty
$$

for $q>2 / \varepsilon$. By Lyapunov's inequality, this means that $\mathrm{E}\left(|\xi|^{q}\right)<+\infty$ for all $q>0$.

Choosing $\eta_{\varepsilon}=C_{H_{\min }, \varepsilon} \xi$ and using (5.2), we prove Lemma 5.2.

Lemma 5.3. Let $1-H_{\min }<\alpha<1$. Then

$$
\begin{aligned}
& \mathrm{E} \sup _{0 \leq t<s \leq T}\left|D_{t+}^{1-\alpha} Y_{t+}(s)\right|^{p}<+\infty, \\
& \mathrm{E} \sup _{0 \leq s<t \leq T}\left|D_{t-}^{1-\alpha} Y_{t-}(s)\right|^{p}<+\infty
\end{aligned}
$$

for all $p>0$.

Proof. The definition of the fractional Weil derivative implies that

$$
\left|D_{t-}^{1-\alpha} Y_{t-}(s)\right| \leq \frac{1}{\Gamma(\alpha)}\left(\frac{\left|Y_{t}-Y_{s}\right|}{(t-s)^{1-\alpha}}+(1-\alpha) \int_{s}^{t} \frac{\left|Y_{y}-Y_{s}\right|}{(y-s)^{2-\alpha}} d y\right) .
$$

According to Lemma 5.2, if $\varepsilon<\alpha-\left(1-H_{\min }\right)$, there exists a random variable $\eta_{\varepsilon}$ whose moments are finite and

$$
\begin{aligned}
\left|D_{t-}^{1-\alpha} Y_{t-}(s)\right| & \leq \frac{\eta_{\varepsilon}}{\Gamma(\alpha)}\left((t-s)^{H_{\min }-\varepsilon-1+\alpha}+(1-\alpha) \int_{s}^{t}(y-s)^{H_{\min }-\varepsilon-2+\alpha} d y\right) \\
& \leq \eta_{\varepsilon} \frac{T^{H_{\min }-\varepsilon-1+\alpha}}{\Gamma(\alpha)}\left(1+\frac{1-\alpha}{H_{\min }-\varepsilon-1+\alpha}\right) .
\end{aligned}
$$

Thus (5.5) follows.

Inequality (5.4) is proved similarly.

Theorem 5.1. Let all the assumptions of Theorem 4.1 hold. Let stochastic processes $Y_{t}^{\varepsilon}$, $\varepsilon>0$, approximate the process $Y_{t}=B_{t}^{H_{t}}$ such that

$$
\left\|Y^{\varepsilon}-Y\right\|_{1, \beta} \stackrel{P}{\rightarrow} 0, \quad \varepsilon \rightarrow 0+,
$$

for some $\beta \in\left(\frac{1}{2}, H_{\min }\right)$. 
Let $X^{\varepsilon}$ be a solution of the equation

$$
X_{t}^{\varepsilon}=X_{0}+\int_{0}^{t} b\left(s, X_{s}^{\varepsilon}\right) d s+\int_{0}^{t} \sigma\left(s, X_{s}^{\varepsilon}\right) d Y_{s}^{\varepsilon}, \quad t \in[0, T] .
$$

Then

$$
\sup _{t \in[0, T]}\left|X_{t}-X_{t}^{\varepsilon}\right| \stackrel{\mathrm{P}}{\rightarrow} 0, \quad \varepsilon \rightarrow 0+.
$$

Proof. It is obvious that

$$
K_{f}:=\sup _{r \in[0, T]} \int_{0}^{r} \frac{\left|f_{r}-f_{s}\right|}{(r-s)^{\beta}} d s \leq C\|f\|_{0,1-\beta} .
$$

The proof of Theorem 5.1 in [7] contains the following bound:

$$
\left\|X^{\varepsilon}\right\|_{0,1-\beta} \leq 2\left(1+\left|X_{0}\right|\right) e^{\lambda_{0}(\varepsilon) T},
$$

where

$$
\begin{gathered}
\lambda_{0}(\varepsilon) \geq\left(2\left(C_{3}+C_{1} \Lambda_{\beta}\left(Y^{\varepsilon}\right)\right)\right)^{1 /(1-2 \beta)}, \\
\Lambda_{\beta}\left(Y^{\varepsilon}\right) \leq\left\|Y^{\varepsilon}\right\|_{1, \beta} .
\end{gathered}
$$

Since

$$
\left\|Y^{\varepsilon}-Y\right\|_{1, \beta} \stackrel{\mathrm{P}}{\rightarrow} 0, \quad \varepsilon \rightarrow 0+,
$$

the random variables $\Lambda_{\beta}\left(Y^{\varepsilon}\right)$ are bounded in probability uniformly with respect to $\varepsilon$. Hence $\left\|X^{\varepsilon}\right\|_{0,1-\beta}$ as well as $K_{X^{\varepsilon}}$ are bounded in probability uniformly with respect to $\varepsilon$. Similarly, $X_{t}^{\varepsilon}$ is bounded in probability uniformly with respect to $\varepsilon$ and $t$. Thus Theorem 5.1 follows from the convergence

$$
\mathrm{P}\left(\sup _{t}\left|X_{t}-X_{t}^{\varepsilon}\right|>\delta, \sup _{t}\left|X_{t}\right| \leq R, \sup _{t}\left|X_{t}^{\varepsilon}\right| \leq R\right) \rightarrow 0, \quad \varepsilon \rightarrow 0+,
$$

for all $\delta>0$ and $R>0$.

The above reasoning allows us to restrict our consideration to the case where $X_{t}$ and $X_{t}^{\varepsilon}$ are bounded by some constant $R>0$.

We have

$$
\begin{gathered}
X=X_{0}+F^{(b)}(X)+G^{(\sigma, Y)}(X), \\
X^{\varepsilon}=X_{0}+F^{(b)}\left(X^{\varepsilon}\right)+G^{\left(\sigma, Y^{\varepsilon}\right)}\left(X^{\varepsilon}\right),
\end{gathered}
$$

where

$$
F^{(b)}(f)(t):=\int_{0}^{t} b(s, f(s)) d s, \quad G^{(\sigma, g)}(f)(t):=\int_{0}^{t} \sigma(\cdot, f(\cdot)) d g
$$

for $t \in[0, T]$. Thus

$$
\begin{aligned}
\left\|X-X^{\varepsilon}\right\|_{0,1-\beta, \lambda} \leq & \left\|F^{(b)}(X)-F^{(b)}\left(X^{\varepsilon}\right)\right\|_{0,1-\beta, \lambda} \\
& +\left\|G^{(\sigma, Y)}(X)-G^{(\sigma, Y)}\left(X^{\varepsilon}\right)\right\|_{0,1-\beta, \lambda}+\left\|G^{\left(\sigma, Y^{\varepsilon}-Y\right)}\left(X^{\varepsilon}\right)\right\|_{0,1-\beta, \lambda} .
\end{aligned}
$$

Applying Propositions 4.2 and 4.4 of [7] we estimate the norm:

$$
\begin{aligned}
\left\|X-X^{\varepsilon}\right\|_{0,1-\beta, \lambda} \leq & C \lambda^{1-2 \beta}\left(1+\|Y\|_{1, \beta}\right)\left(1+K_{X}+K_{X_{\varepsilon}}\right)\left\|X-X^{\varepsilon}\right\|_{0,1-\beta, \lambda} \\
& +C \lambda^{1-2 \beta}\left\|Y-Y^{\varepsilon}\right\|_{1, \beta}\left(1+\left\|X^{\varepsilon}\right\|_{0,1-\beta, \lambda}\right) .
\end{aligned}
$$

If

$$
\Theta(\lambda, \varepsilon)=C \lambda^{1-2 \beta}\left\{\left(1+\|Y\|_{1, \beta}\right)\left(1+K_{X}+K_{X_{\varepsilon}}\right)+\left\|X^{\varepsilon}\right\|_{0,1-\beta, \lambda}\right\}<\frac{1}{2},
$$


then

This yields

$$
\left\|X-X^{\varepsilon}\right\|_{0,1-\beta, \lambda} \leq\left\|Y-Y^{\varepsilon}\right\|_{1, \beta} .
$$

$$
\mathrm{P}\left(\left\|X-X^{\varepsilon}\right\|_{0,1-\beta, \lambda}>c\right) \leq \mathrm{P}\left(\left\|Y-Y^{\varepsilon}\right\|_{1, \beta}>c\right)+\mathrm{P}\left(\Theta(\lambda, \varepsilon)>\frac{1}{2}\right) .
$$

Note that $\sup _{t \in[0, T]}\left|X_{t}-X_{t}^{\varepsilon}\right| \leq e^{\lambda T}\left\|X-X^{\varepsilon}\right\|_{0,1-\beta, \lambda}$, whence

$$
\mathrm{P}\left(\sup _{t \in[0, T]}\left|X_{t}-X_{t}^{\varepsilon}\right|>\delta\right) \leq \mathrm{P}\left(\left\|Y-Y^{\varepsilon}\right\|_{1, \beta}>\delta e^{-\lambda T}\right)+\mathrm{P}\left(\Theta(\lambda, \varepsilon)>\frac{1}{2}\right)
$$

for an arbitrary $\delta>0$.

On the other hand, it is easy to show that $\Theta(\lambda, \varepsilon) \rightarrow 0$ in probability as $\lambda \rightarrow \infty$ uniformly with respect to $\varepsilon$.

\section{Concluding Remarks}

We considered approximations of multifractional Brownian motion by absolutely continuous processes. We proved a theorem on the existence and uniqueness of a solution for stochastic differential equations with multifractional Brownian motion. We constructed an approximation of solutions of such equations by solutions of ordinary stochastic differential equations.

\section{BIBLIOGRAPHY}

1. R. J. Adler, An Introduction to Continuity, Extrema, and Related Topics for General Gaussian Processes, IMS Lecture Notes-Monograph Series, vol. 12, Institute of Mathematical Statistics, Hayward, CA, 1990. MR1088478 (92g:60053)

2. A. Benassi, S. Jaffard, and D. Roux, Gaussian processes and pseudodifferential elliptic operators, Revista Mathematica Iberoamericana 13 (1997), no. 1, 19-89. MR1462329 (98k:60056)

3. B. Boufoussi, M. Dozzi, and R. Marty, Local time and Tanaka formula for a Volterra-type multifractional Gaussian process, http://hal.archives-ouvertes.fr/hal-00389740/en/. Bernoulli 16 (2010), no. 4, 1294-1311. MR2759180

4. S. Cohen, From self-similarity to local self-similarity: The estimation problem, Fractals: Theory and Applications in Engineering, Springer, London, 1999, pp. 3-16. MR1726364 (2001c:60063)

5. A. M. Garsia, E. Rodemich, and H. Rumsey Jr., A real variable lemma and the continuity of paths of some Gaussian processes, Indiana Univ. Math. J. 20 (1970), no. 6, 565-578. MR0267632 (42:2534)

6. Yu. Mishura, Stochastic Calculus for Fractional Brownian Motion and Related Processes, Lecture Notes in Mathematics, vol. 1929, Springer, Berlin, 2008. MR.2378138 (2008m:60064)

7. D. Nualart and A. Răşcanu, Differential equations driven by fractional Brownian motion, Collect. Math. 53 (2002), no. 1, 55-81. MR.1893308 (2003f:60105)

8. R. F. Peltier and J. Lévy Véhel, Multifractional Brownian motion: definition and preliminary results, INRIA research report, vol. 2645, 1995.

9. K. V. Ral'chenko and G. M. Shevchenko, Properties of the paths of a multifractal Brownian motion, Teor. Imovir. Mat. Stat. 80 (2009), 106-116; English transl. in Theory Probab. Math. Statist. 80 (2010), 119-130. MR2541957(2010h:60122)

10. S. G. Samko, A. A. Kilbas, and O. I. Marichev, Fractional integrals and derivatives. Theory and applications, Nauka i tekhnika, Minsk, 1987; English transl., Gordon and Breach Science Publishers, Yverdon, 1993. MR1347689 (96d:26012)

Department of Probability Theory, Statistics, and Actuarial Mathematics, Faculty for Mechanics and Mathematics, National Taras Shevchenko University, Academician Glushkov Avenue 2, Kiev 03127, Ukraine

E-mail address: k.ralchenko@gmail.com

Received 8/DEC/2009

Translated by N. SEMENOV 